\title{
Risk of multiple myeloma is associated with polymorphisms within telomerase genes and telomere length
}

\author{
Daniele Campa ${ }^{1 \star}$, Alessandro Martino ${ }^{2 \star}$, Judit Varkonyi ${ }^{3}$, Fabienne Lesueur ${ }^{4}$, Krzysztof Jamroziak ${ }^{5}$, Stefano Landi ${ }^{6}$, \\ Artur Jurczyszyn ${ }^{7}$, Herlander Marques ${ }^{8,9}$, Vibeke Andersen ${ }^{10,11}$, Manuel Jurado ${ }^{12,13}$, Hermann Brenner ${ }^{14,15}$, Mario Petrini ${ }^{16}$, \\ Ulla Vogel ${ }^{17}$, Ramón García-Sanz ${ }^{18}$, Gabriele Buda ${ }^{16}$, Federica Gemignani ${ }^{6}$, Rafael Ríos ${ }^{12,13}$, Annette Juul Vangsted ${ }^{19}$, \\ Charles Dumontet ${ }^{20}$, Joaquín Martínez-López ${ }^{21}$, María José Moreno ${ }^{22}$, Anna Stępień ${ }^{23}$, Marzena Wątek ${ }^{24}$, Victor Moreno ${ }^{25}$, \\ Aida Karina Dieffenbach ${ }^{14,15}$, Anna Maria Rossi ${ }^{6}$, Katja Butterbach ${ }^{14}$, Svend E. Hove Jacobsen ${ }^{26}$, Hartmut Goldschmidt ${ }^{27}$, \\ Juan Sainz ${ }^{12,13}$, Jens Hillengass ${ }^{27}$, Enrico Orciuolo ${ }^{16}$, Marek Dudziński ${ }^{28}$, Niels Weinhold ${ }^{27}$, Rui Manuel Reis ${ }^{8,9,29}$ \\ and Federico Canzian ${ }^{2}$ \\ ${ }^{1}$ Division of Cancer Epidemiology, German Cancer Research Center (DKFZ), Heidelberg, Germany \\ ${ }^{2}$ Genomic Epidemiology Group, German Cancer Research Center (DKFZ), Heidelberg, Germany \\ ${ }^{3}$ Department of Internal Medicine, Semmelweis University, Budapest, Hungary \\ ${ }^{4}$ Genetic Epidemiology of Cancer Team, INSERM, Ugoo, Institut Curie, Mines ParisTech, Paris, France \\ ${ }^{5}$ Department of Hematology, Medical University of Łodz, Łodz, Poland \\ ${ }^{6}$ Department of Biology, University of Pisa, Pisa, Italy \\ ${ }^{7}$ Department of Hematology, Cracow University Hospital, Cracow, Poland \\ ${ }^{8}$ Life and Health Sciences Research Institute, University of Minho, Braga, Portugal \\ ${ }^{9}$ ICVS/3B's-PT Government Associate Laboratory, University of Minho, Braga/Guimarães, Portugal \\ ${ }^{10}$ Organ Center, Hospital of Southern Jutland, Aabenraa, Denmark \\ ${ }^{11}$ Institute of Regional Health Research, Faculty of Health Sciences, University of Southern Denmark, Odense C, Denmark \\ ${ }^{12}$ Genomic Oncology Area, GENYO, Centre for Genomics and Oncological Research, Pfizer/University of Granada/Andalusian Regional Government, \\ Granada, Spain \\ ${ }^{13}$ Hematology Department, Virgen de las Nieves University Hospital, Granada, Spain \\ ${ }^{14}$ Division of Clinical Epidemiology and Aging Research, German Cancer Research Center (DKFZ), Heidelberg, Germany \\ ${ }^{15}$ German Cancer Consortium (DKTK), Heidelberg, Germany \\ ${ }^{16}$ UO Hematology, Department of Internal and Experimental Medicine, University of Pisa, Pisa, Italy \\ ${ }^{17}$ National Research Centre for the Working Environment, Copenhagen, Denmark \\ ${ }^{18}$ Department of Hematology, University Hospital of Salamanca, Salamanca, Spain \\ ${ }^{19}$ Department of Hematology, Roskilde Hospital, Copenhagen University, Roskilde, Denmark \\ ${ }^{20}$ INSERM UMR 1052/CNRS 5286, Université Claude Bernard Lyon I, Lyon, France \\ ${ }^{21}$ Department of Hematology, Hospital Universitario Doce de Octubre, Madrid, Spain \\ ${ }^{22}$ Department of Hematology, Morales Meseguer General University Hospital, Murcia, Spain \\ ${ }^{23}$ Laboratory of Clinical and Transplant Immunology and Genetics, Copernicus Memorial Hospital, Łodz, Poland \\ ${ }^{24}$ Hematology Clinic, Holycross Cancer Center, Kielce, Poland \\ ${ }^{25}$ Programme of Cancer Prevention and Unit of Biomarkers and Susceptibility, IDIBELL-Catalan Institute of Oncology and University of Barcelona, Barce- \\ lona, Spain \\ ${ }^{26}$ Clinic of Immunology, Laboratory Center, Hospital of Southern Jutland, Sønderborg, Denmark \\ ${ }^{27}$ Department of Internal Medicine V, University of Heidelberg, Heidelberg, Germany \\ ${ }^{28}$ Division of Hematology, Rzeszow Regional Hospital, Rzeszow, Poland \\ ${ }^{29}$ Molecular Oncology Research Center, Barretos Cancer Hospital, Barretos, Brazil
}

Key words: multiple myeloma, polymorphisms, telomere length, telomerase, susceptibility Additional Supporting Information may be found in the online version of this article.

${ }^{*}$ D.C. and A.M. contributed equally to this work.

Grant sponsor: Polish Ministry of Science and Higher Education; Grant number: NN402178334; Grant sponsors: Research Fund at Region Sjælland, DK; Baden-Württemberg State Ministry of Science, Research and Arts; CRIS Foundation

DOI: $10.1002 / \mathrm{ijc} .29101$

History: Received 14 May 2014; Accepted 23 June 2014; Online 28 Jul 2014

Correspondence to: Federico Canzian, Genomic Epidemiology Group (C055), German Cancer Research Center/Deutsches Krebsforschungszentrum (DKFZ), Im Neuenheimer Feld 280, 69120 Heidelberg, Germany, Tel.: +49-6221-421791, Fax: +49-6221-421810, E-mail: f.canzian@dkfz.de 
Compelling biological and epidemiological evidences point to a key role of genetic variants of the TERT and TERC genes in cancer development. We analyzed the genetic variability of these two gene regions using samples of 2,267 multiple myeloma (MM) cases and 2,796 healthy controls. We found that a TERT variant, rs2242652, is associated with reduced MM susceptibility ( $\mathrm{OR}=0.81 ; 95 \% \mathrm{Cl}: 0.72-0.92 ; p=0.001)$. In addition we measured the leukocyte telomere length (LTL) in a subgroup of 140 cases who were chemotherapy-free at the time of blood donation and 468 controls, and found that MM patients had longer telomeres compared to controls $\left(\mathrm{OR}=1.19 ; 95 \% \mathrm{Cl}: 0.63-2.24 ; p_{\text {trend }}=0.01\right.$ comparing the quartile with the longest $\mathrm{LTL}$ versus the shortest LTL). Our data suggest the hypothesis of decreased disease risk by genetic variants that reduce the efficiency of the telomerase complex. This reduced efficiency leads to shorter telomere ends, which in turn may also be a marker of decreased MM risk.

\section{What's new?}

A critical element of cancer cell immortality is the maintenance of telomere length, a process that is influenced in part by genetic variations in telomerase reverse transcriptase (TERT) and telomerase RNA component (TERC). At the TERT locus in particular, certain variations are linked with either increased or decreased risk of a variety of malignancies. In the present study, a variant of TERT known as rs2242652 was associated with reduced risk of multiple myeloma. Compared with controls, patients with multiple myeloma were found to possess longer telomeres, suggesting an association between increased telomere length and increased multiple myeloma risk.

Multiple myeloma (MM) is the second most common hematological cancer and it arises from a single clone of malignant plasma cells (PCs) in the bone marrow. ${ }^{1}$ In the most advanced stages of the disease, malignant PCs can subsequently migrate in extra-medullary districts. The mean age at diagnosis is usually around 60 years, ${ }^{1}$ while the worldwide incidence of this neoplasm is around 1.5/100,000 new cases (age-standardized ratio). In Europe the incidence is slightly higher, with 4.6/ 100,000 and 3.2/100,000 new cases, respectively in men and women. Risk factors for MM have been reviewed in Martino et $a .^{2} \mathrm{MM}$ risk is clearly related to age, gender, ethnicity and the presence of pre-malignant conditions such as MGUS. There are epidemiological evidences supporting an increased risk of MM among obese people and for those who have a low intake either of fish or vegetables. Other suggested risk factors are autoimmune diseases, viral infections, exposures to pesticides, organic solvents, hairdresser's products, smoking and alcohol consumption. However the evidences are largely inconsistent.

Multiple lines of evidence suggest that genetic factors are involved in MM pathogenesis. ${ }^{3,4}$ Several risk loci have been proposed and a few have been identified through genomewide association studies (GWAS). ${ }^{2,5-11}$

The genetic variability of the telomerase reverse transcriptase (TERT) and the telomerase RNA component (TERC) gene regions could play a role in MM etiology for two reasons: first the two genes are responsible for crucial cellular processes, namely telomere homeostasis, second their polymorphic variants have been found to be associated with multiple cancer types and other human phenotypes. ${ }^{12-24}$

TERT and TERC constitute the telomerase complex, ${ }^{25}$ whose correct functioning is fundamental for the accurate de novo synthesis of telomeric ends. Even moderate changes in TERT and TERC activity could profoundly affect telomere homeostasis. ${ }^{26,27}$ Telomeres are highly specialized structures that have a key role in various cellular processes such as chromosomal stability and cell growth ${ }^{28-30}$ and in the proper segregation of chromosomes to daughter cells. ${ }^{31}$ There is overwhelming evidence suggesting telomere dysfunction, mediated by telomerase activation, as a driving force in cancer development. ${ }^{26,32,33}$

TERT is a pleiotropic gene with several regulatory functions other than catalyzing the synthesis of the telomeric ends, including transcriptional regulation, subtelomeric silencing and stem cell mobilization. ${ }^{30}$ It is not, therefore, surprising that it harbors multiple variants associated with risk of different diseases and in particular with various cancer types. For example the rs2736100 SNP is associated with glioma, testicular and lung cancer, ${ }^{16,18,20}$ while rs401681 is associated with lung, bladder and pancreatic cancer, ${ }^{17,19,21}$ rs10069690 with estrogen receptor-negative breast cancer ${ }^{12,13}$ and rs2242652 with breast, prostate and ovarian cancer. ${ }^{12,14,15}$ In addition, TERT polymorphisms are associated with other human phenotypes such as pulmonary fibrosis and PSA levels. ${ }^{22-24,34}$

A very recent GWAS has reported the association of rs10936599 with increased risk of MM. ${ }^{7}$ This polymorphism lies in $3 q 26.2$, in a linkage disequilibrium (LD) region that includes also the TERC gene. Clearly this finding further increases the a priori hypothesis of the involvement of the two genes in the disease.

Moreover polymorphic variants in both TERT and TERC have been consistently associated with telomere length measured in leukocytes (LTL), ${ }^{35,36}$ which in turn is a putative 
marker for cancer incidence and progression. In the last years telomere length (TL) has been intensively studied in relation to risk and survival of various cancer types, including hematological ones. ${ }^{5,26,37,38}$ In particular in a very recent study performed within the European prospective investigation on cancer (EPIC), Hosnijeh et al. ${ }^{39}$ have shown that longer telomeres are associated with increased risk of MM. In most epidemiological studies TL is measured in DNA from peripheral blood and used as a proxy for TL in other tissues. Whether LTL reflects TL of other tissues is a matter of debate. Nevertheless, it has been shown that LTL correlates strongly with TL in hematopoietic stem cells and hematopoietic progenitor cells both in newborn and adults. ${ }^{40}$ In addition, the inter-cell type variability is remarkably lower than the inter-individual variability of LTL. ${ }^{40}$ Therefore, at least for the hematopoietic cell lineages, it is reasonable to assume that LTL is an accurate proxy of TL in other cell subtypes.

Thus there is compelling biological and epidemiological evidence that point to a key importance of the TERT and TERC genetic variants in cancer development, either as mediators of TL, or through other not yet elucidated mechanisms. Detailed genetic analysis of the TERT region has been performed for some solid tumors, ${ }^{15}$ but no such effort has been done in hematologic cancers.

Following these leads we have performed an in-depth analysis of the genetic variability of the two regions. We have used, in a two-phase study, biological material of 2,267 MM cases and 2,796 healthy controls in the context of the Heidelberg Multiple Myeloma Group, the ESTHER cohort and the International Multiple Myeloma rESEarch (IMMEnSE) consortium. In addition we have also measured LTL in $140 \mathrm{MM}$ cases and 468 controls, in order to investigate, for the first time in a retrospective study of MM, if LTL is a susceptibility marker for the disease. This effort is of particular importance because it can link the genetic variability (the TERC and TERT SNPs) and an intermediate phenotype (telomere length) to the disease.

\section{Material and Methods \\ Study populations and study design}

We used three populations: the Heidelberg MM Group, the ESTHER cohort and the IMMEnSE consortium. The first two studies were used as discovery set (phase I), respectively using German MM patients from the Heidelberg MM Group and German healthy controls from the ESTHER study. The IMMEnSE consortium population has been used as a replication set (phase II) of the most significant associations found in phase I.

The Heidelberg MM Group patients comprised 585 cases collected by the University Hospital in Heidelberg (Southwest of Germany). In the ESTHER study 9,953 participants were recruited in a 2-year span between July 2000 and December 2002 in Saarland (a state in Southwest Germany). The participants were aged 50-75 years at enrollment $($ mean $=62.1$; $\mathrm{SD}=6.6$ ). Participants were recruited by their general practi-
Table 1. Description of the study population

\begin{tabular}{|c|c|c|c|}
\hline & Cases & Controls & Total \\
\hline \multicolumn{4}{|c|}{ Geographic origin } \\
\hline Germany $^{1}$ & 585 & 902 & 1,487 \\
\hline Italy ${ }^{2}$ & 258 & 237 & 495 \\
\hline Portugal $^{2}$ & 68 & 99 & 167 \\
\hline Spain $^{2}$ & 319 & 427 & 746 \\
\hline France $^{2}$ & 127 & 191 & 318 \\
\hline Hungary $^{2}$ & 159 & 105 & 264 \\
\hline Poland ${ }^{2}$ & 433 & 320 & 753 \\
\hline Denmark $^{2}$ & 318 & 515 & 833 \\
\hline Total & 2,267 & 2,796 & 5,063 \\
\hline \multicolumn{4}{|l|}{ Gender } \\
\hline Males & $52 \%$ & $49 \%$ & \\
\hline Females & $48 \%$ & $51 \%$ & \\
\hline \multicolumn{4}{|c|}{ Median age $(25-75 \% \text { percentiles })^{3}$} \\
\hline IMMEnSE & $61(54-68)$ & $55(44-65)$ & \\
\hline Germany ${ }^{2}$ & $56(50-61)$ & $61(56-66)$ & \\
\hline
\end{tabular}

${ }^{1}$ Cases from the Heidelberg MM Group, controls from the ESTHER cohort (phase I).

${ }^{2}$ Subjects from the IMMEnSE consortium (phase II).

${ }^{3} \mathrm{Age}$ at diagnosis for cases, age at recruitment for controls.

tioners during a general health check-up. Eligible subjects who accepted to participate in the study completed a questionnaire and provided a blood sample. In this study, a subsample of 902 ESTHER subjects was used in the analysis of the discovery phase.

The second phase of the study was conducted in the context of the International Multiple Myeloma rESEarch (IMMEnSE) consortium. ${ }^{2}$ The IMMEnSE study population used for this study consisted of $1682 \mathrm{MM}$ cases and 1894 controls recruited from seven different European countries. Briefly, cases were defined by a confirmed diagnosis of MM according to the International Myeloma Working Group (IMWG) criteria. $^{41}$ Different region-specific subpopulations of controls were selected among the general population as well as among hospitalized subjects with different diagnoses excluding cancer. Written informed consent was obtained from each participant.

Relevant information on the study population is given in Table 1.

\section{SNP selection}

The common genetic variability of the TERT gene regions was investigated following a tagging approach and adding to the selection SNPs previously shown to be associated with cancer risk or telomere length. All SNPs within the region of TERT/CLPTM1L (chr5:1277490-1377121, NCBI36/hg18) with a minor allele frequency (MAF) $>5 \%$ in Caucasians (International HapMapProject, version 28; http://www.hapmap.org) were included. Tagging SNPs were selected with the 
use of the Haploview Tagger Program (http://www.broad.mit. edu/mpg/haploview/; http://www.broad.mit.edu/mpg/tagger/), using pairwise tagging with a minimum $r^{2}$ of 0.8 . We selected additional SNPs significantly associated at a genome-wide level with cancer risk or with telomere length. ${ }^{12,15}$ For the TERC gene we selected SNPs that have been previously associated with telomere length or cancer risk and that reside in chr3:170974797-170984874 (NCBI36/hg18). ${ }^{35,36,42}$ The final selection consisted of 29 SNPs, 22 in the TERT region and 7 in TERC.

\section{Genotyping}

Genomic DNA was extracted from peripheral blood and was stored frozen at $-20^{\circ} \mathrm{C}$. Genotyping was carried out at the German Cancer Research Center (DKFZ) in Heidelberg using TaqMan (ABI, Applied Biosystems, Foster City, CA) and KASPar (LGC Genomics/KBioscence, Hoddesdon, UK) technologies, according to the protocol specified by the manufacturers and using respectively as master mixes the HOT FIREPol (Solis Biodyne, Tartu, Estonia) and the KASPar indirect assay reagent (LGC Genomics/KBioscence). The order of DNAs from cases and controls was randomized on 384 well plates in order to ensure that a similar number of cases and controls were analyzed simultaneously in the same plate. For quality control purpose, duplicates of $10 \%$ of the samples were interspersed throughout the plates. PCR reactions were performed using a Hydrocycler instrument (LGC Genomics/KBioscence) and the GeneAmp® PCR System (Applied Biosystems).

PCR plates were read on a ViiA7 real time instrument (Applied Biosystems). The ViiA7 RUO Software, version 1.2.2 (Applied Biosystems) was used to determine the genotypes.

\section{Leukocyte telomere length measurement}

Genomic DNA to measure telomere length was isolated from cases and controls. For the cases the starting material for DNA extraction was mononuclear cells separated from peripheral blood while for the controls it was whole blood. The average LTL was estimated in 585 cases from the Heidelberg MM group and a subsample of 468 randomly selected controls from the ESTHER cohort. LTL was measured by real-time PCR quantitative analysis (qPCR), measuring the number of copies of telomeric repeats compared to a single copy gene (RLPO), used as a quantitative control. ${ }^{43}$ Two separate plates were used for PCR reactions: one contained primers specifically amplifying the telomeres (T), while the other primers amplify a single copy gene (S). In addition, for each plate a standard was prepared, by using a reference DNA sample diluted in series, in order to produce five concentrations of DNA (range: $0.468-30 \mathrm{ng} \mu \mathrm{l}^{-1}$ ). Each PCR well contained $10 \mathrm{ng}$ of DNA, $10 \mu \mathrm{l}$ of mix containing the primers specific for each plate $\mathrm{T}$ and $\mathrm{S}, \mathrm{PCR}$ reagents and SYBR green dye for fluorescence detection. Finally, the relative length of telomeres $(\mathrm{T})$ compared with the control gene
(S) was calculated as the ratio T/S. All the samples were analyzed in triplicate. For each measurement the mean of the two closest values out of three was used. Supporting Information Table 4 includes primer sequences used for the amplification of $\mathrm{T}$ and $\mathrm{S}$, and details on the PCR amplification protocols.

\section{Statistical analysis}

The Hardy-Weinberg equilibrium (HWE) was tested in controls for each polymorphism. In the first phase we used 585 cases and 902 healthy controls of German origin to perform an unconditional logistic regression to assess the main effects of the 29 selected genetic polymorphisms on MM risk using a co-dominant and a dominant inheritance model, calculating odds ratio (OR) and confidence intervals (CI). For each SNP the more common allele in the controls was assigned as the reference category. All analyses were adjusted for age (continuous), gender and region of origin. Given the strong a priori probability to find a significant association, all SNPs showing association with MM risk with $p<0.15$ were genotyped in an additional set of 1,682 MM cases and 1,894 controls recruited from the IMMEnSE consortium. This selection resulted in eight SNPs in TERT and one in TERC. In addition, SNP rs10936599 was added to phase two due to the association with MM risk reported in a recent GWAS. The obtained genotyping data were pooled with the ones obtained from phase one of the study and an unconditional logistic regression using the same criteria as described above was carried out. The significance threshold of the final analysis was adjusted, taking into account the large number of tests carried out. We used a modified Bonferroni adjustment that takes into account the effective number of independent variables tested in the model. Because SNPs that are in linkage disequilibrium reflect at least partially the same information, to take into account the possibility of residual LD between the SNPs selected in this study, for each locus we calculated the effective number of independent SNPs, (which is called $M_{\mathrm{eff}}$ ), using the SNP Spectral Decomposition approach (simpleM method). ${ }^{44}$ We obtained a study-wide $M_{\text {eff }}$ value, by adding up the individual gene $M_{\text {eff }}$. The study-wise $M_{\text {eff }}$ obtained was 18. Thus, the threshold for statistical significance was $0.0028(0.05 / 18)$. For SNP rs10936599 we also conducted a meta-analysis with the results of the study by Chubb et al. ${ }^{7}$ reaching a total sample size of 6,112 $\mathrm{MM}$ cases and 12,905 healthy controls. Finally we also performed a meta-analysis with the results of Hosnijeh et al. ${ }^{39}$ to better determine the effect of LTL and MM risk.

Unconditional logistic regression was used to obtain the OR and $95 \%$ CI for the strength of the association between LTL and risk of MM. LTL was analyzed as a categorical variable, using quartiles according to its distribution in control subjects, with the lowest quartile as the referent. All the analyses were conducted with STATA software (StataCorp, College Station, TX). 




\section{Bioinformatic analysis}

We used several bioinformatic tools to assess possible functional relevance of rs2242652 that showed the most significant association with risk of MM. RegulomeDB (http:// regulome.stanford.edu $/)^{45}$ and HaploReg v2B ${ }^{46}$ were used to identify the regulatory potential of the region nearby the SNP. Genevar (http://www.sanger.ac.uk/resources/software/ genevar/) ${ }^{47}$ and GTex (http://commonfund.nih.gov/GTEx/ index) were used to identify potential associations between the SNP and expression levels of nearby genes (eQTL).

\section{Results}

All the analyzed SNPs were in HWE in controls. The genotyping concordance rate between duplicate quality controls was higher than $99 \%$ and the average SNP call rate was 98.37\%.

\section{SNP main effect}

We performed a two step analysis analyzing 29 SNPs in a discovery phase and replicating the best associations (9 SNPs that showed association with MM risk with $p<0.15$ in the first phase) in a larger sample size, for a total of 2,267 MM cases and 2,796 healthy controls. In addition a susceptibility locus (SNP rs10936599) that lies in the TERC region and was recently reported in a GWAS on MM risk was added to the second phase. Results of the first phase are reported in Supporting Information Tables 1 and 2, while results of the joint analysis of phase 1 and 2 are reported in Table 2. Supporting Information Table 7 reports results of SNP and MM risk analysis stratified by country. We found that 1 SNP was associated with MM risk considering the threshold for statistical significance to be 0.0028 and a second variant was very close to statistical significance. The most significant association was between the minor allele of the TERT polymorphic variant rs2242652 and a decreased risk of $\mathrm{MM}\left(\mathrm{OR}_{\text {dominant }}=0.81 ; 95 \% \mathrm{CI}\right.$ $0.72-0.92 ; p=0.001)$. The other association was between the TERC SNP rs12696304 and decreased risk of MM $\left(\mathrm{OR}_{\text {dominant }}=0.83 ; 95 \%\right.$ CI $\left.0.74-0.94 ; p=0.003\right)$. In addition we found one polymorphic variant that was associated with MM risk at the conventional 0.05 threshold. Specifically the TERT SNP rs10069690 was associated with decreased risk of $\mathrm{MM}\left(\mathrm{OR}_{\text {dominant }}=0.86\right.$; CI $0.75-0.99$; $p=0.03$ ). However this SNP is in linkage disequilibrium with rs2242652 $\left(r^{2}=0.82\right.$ in 1000G) and therefore they reflect the same association. Finally, rs10936599 did not show a significant association with MM risk in the first phase of the study (Supporting Information Table 2), but the association was observed in the IMMenSE population $\left(\mathrm{OR}_{\text {dominant }}=0.83 ; 95 \%\right.$ CI $\left.0.71-0.99 ; p=0.037\right)$ and it reached study-wide statistical significance in the joint analysis $\left(\mathrm{OR}_{\text {dominant }}=0.82 ; 95 \%\right.$ CI $\left.0.72-0.93 ; p=0.002\right)$. The two polymorphisms in the TERC region are in strong LD $\left(r^{2}=0.82\right)$, indicating that the two associations found are not independent and represent the same locus. 
Table 3. Associations between LTL and MM risk

\begin{tabular}{|c|c|c|c|c|c|}
\hline Relative LTL $^{1}$ & Controls/cases & $O R^{2}$ & $95 \% \mathrm{Cl}^{3}$ & $p_{\text {value }}$ & $p_{\text {trend }}$ \\
\hline Quartile $1(0.24-0.78)$ & $125 / 22$ & Ref. & - & - & 0.011 \\
\hline Quartile $2(0.79-0.91)$ & $105 / 33$ & 1.48 & $0.78-2.83$ & 0.232 & \\
\hline Quartile 3 (0.92-1.09) & $119 / 40$ & 1.14 & $0.60-2.17$ & 0.680 & \\
\hline Quartile 4 (1.09-1.95) & $119 / 45$ & 1.19 & $0.63-2.24$ & 0.588 & \\
\hline
\end{tabular}

${ }^{1}$ Cases recruited in Heidelberg who were chemotherapy-free at the time of blood collection $(N=140) v s$. healthy controls $(N=468)$.

${ }^{2} \mathrm{OR}=$ odds ratio, adjusted by age and gender.

${ }^{3} 95 \%$ Confidence interval, adjusted by age and gender.

\section{Meta-analysis}

For SNP rs10936599 we also performed a meta-analysis between our results and those from Chubb et al., ${ }^{7}$ including a total of 6,112 MM cases and 12,905 healthy controls. For the meta-analysis all the cases from the Heidelberg MM group were excluded due to the partial overlap with the publication by Chubb. The results are conclusive indicating a highly statistically significant protective effect of the minor allele of the SNP $(\mathrm{OR}=0.80 ; 95 \%$ CI $0.76-0.85 ; p=4.2 \times$ $\left.10^{-14}\right)$.

\section{Effect of SNPs on LTL}

We also attempted to correlate the selected SNPs with LTL and we found that the minor alleles of the rs10936599 and rs12696304 SNPs were associated, at the conventional threshold of $p<0.05$, with shorter telomeres (beta $=-0.093$; $p=0.026$; and beta $=-0.075 ; p=0.031$, respectively). In addition we observed that several SNPs reported in the literature to be associated with LTL, such as rs7726159, rs2736100 and rs7705526 showed a similar effect in our study, although they did not reach statistical significance. The complete results for each SNP are shown in Supporting Information Table 5.

\section{LTL and MM risk}

We calculated crude and adjusted (gender and age) OR. Chemotherapeutic treatment and sample handling could modify telomere length and therefore we considered as reliable only the cases $(N=140)$ who were chemotherapy-free at the time of blood drawing and whose samples were handled homogeneously in terms of time from blood collection to DNA isolation and DNA extraction method. We found a weak association of longer TL with increased risk of MM $\left(p_{\text {trend }}=0.01\right)$. The complete results are shown in Table 3 . Furthermore, as an exploratory analysis, we considered also the patients who were recruited outside Heidelberg and those who had already received chemotherapy at the time of blood collection. The results show a considerable degree of heterogeneity, but they all point to an association between longer telomeres and MM risk (Supporting Information Table 3). We also performed a meta-analysis with results of a recent report on LTL and MM risk performed in the EPIC study. The results of that study clearly suggest an association between longer LTL and increased risk of MM. We have meta-analyzed the results form Hosnijeh and ours considering all the cases together and only the subgroup recruited in Heidelberg and chemotherapy free at the time of blood collection. The results of the meta-analysis are shown in Supporting Information Table 6. The meta-analysis, as expected, confirmed the observation that longer telomere are associated with an increased MM risk.

We found a highly statistically significant inverse correlation between age and LTL (beta $=-0.0082 ; p=2.21 \times$ $\left.10^{-11}\right)$.

\section{Possible functional effects}

In the Genevar database the rs2242652 SNP is not present. RegulomeDB showed a score of 5, indicating the possible presence of a transcription factor binding motif or a DNase sensitivity peak. HaploReg suggested the presence of DNase sensitivity peak in several cell types and the possible alteration of nine regulatory motives.

\section{Discussion}

With our comprehensive analysis of the TERT and the TERC loci we have confirmed a recent GWAS signal in TERC and identified a novel association between a TERT SNP and MM risk. In addition we have observed an association between long LTL and increased MM risk.

We confirmed the association of the minor allele of SNP TERC-rs10936599 with a decreased risk of MM, providing a strong evidence of this locus in the disease etiology. We also genotyped another variant (rs12696304) in the same gene region, that is in LD with TERC-rs10936599. The two SNPs are close to each other $(10,830 \mathrm{bp})$ and they are highly correlated $\left(r^{2}=0.82\right)$ in our population. Both TERC polymorphisms have a functional relevance since they have been consistently associated with telomere length. ${ }^{35,36,42}$ However it is not clear yet whether one of the two variants is the real etiological cause for $\mathrm{MM}$ or if they are both linked to a yet unknown polymorphic variant.

From a genetic standpoint the most novel finding of this study is the association of the minor allele of rs2242652 and a decreased risk of MM. This polymorphism lies in intron four of the TERT gene and it is a pleiotropic SNP since it has been consistently associated with breast, prostate and skin cancer risk. ${ }^{12,14}$ As is the case when a tagging approach is selected, it is difficult to determine whether the 
polymorphic variant found associated in this study is causal or is just a disease marker, however there are several clues that seem to indicate the effective involvement of rs2242652 in cancer risk. It lies in a DNase I hypersensitive region, a stretch of chromatin which is characterized by an intense transcriptional activity and that is accessible to transcription factors. ${ }^{15}$ The results of our analysis using Genevar, RegulomeDB and HaploReg taken together do not convincingly identify a mechanistic link between the SNP and the function of TERT or nearby genes. However Kote-Jarai et al. have reported that the minor allele of rs2242652 is associated with a decreased TERT expression and also that it is predicted to inhibit the binding of several transcription factors, ${ }^{15}$ an observation that would be consistent with a decreased gene expression. In an independent study Bojesen et al. found that the minor alleles of rs2242652 and rs10069690, respectively, increase silencing and generate a truncated TERT splice variant, ${ }^{12}$ an observation that, again, is consistent with a down-regulatory effect of the polymorphism on the gene expression and activity.

It is difficult to answer the question whether longer or shorter telomeres are associated with cancer risk since there is much heterogeneity in the results of association studies. ${ }^{5}$ In this study we found a weak tendency of MM cases having longer telomeres than controls, an observation that is in line with the latest results on other organs. Since it has been recently shown that differences in sample preparation can have a large impact on LTL measurements ${ }^{12}$ we decided to consider only cases collected in an homogeneous way (i.e., recruited locally before chemotherapy). Although we observed a large degree of heterogeneity according to whether cases had received chemotherapy before or after blood drawing and according to the origin of cases, all subgroups analyses point to an association between longer telomeres and MM risk. Considering the small sample size we could use, our results on LTL should be taken with caution, although if replicated in independent studies, they could clearly link the genetic variability of the selected regions to MM risk.

Indeed, both polymorphic variants found in our study are functional and lie in genes which catalyze de novo addition of telomeric repeats onto chromosome ends and, therefore, it seems logical that telomere length might be the connection between genetic variability and increased MM risk. In particular a down-regulation of the TERT gene should produce shorter telomere ends. Our observation that the minor allele of rs2242652 is associated with decreased MM risk and the previous reports that it down-regulates TERT expression, and therefore causes shorter TL, is consistent with our finding that longer LTL is, instead, associated with increased MM risk. Additionally in a very recent manuscript Hosnijeh et al. ${ }^{39}$ explored the possible association between LTL and lymphoma risk in the context of the EPIC study. Among the cases used in that study there was also a small subgroup of MM (107 cases). The results found by Hosnijeh et al. are in complete agreement with what we find, namely that longer telomere are associated with increased MM risk increasing the strength of our observations. The meta-analysis that we have performed further corroborates these findings.

We also found that several polymorphic variants are associated with LTL, although considering the correction for multiple testing none of these associations remained significant. It is however interesting to note that we were able to replicate several known associations between SNPs and LTL, such as the association of the minor rs10936599 allele with shorter telomeres, with an estimate almost super-imposable to what was reported by Codd et al. in a very large study. ${ }^{35}$ We did not observe an association for rs2242652 on LTL; however this effect was seen by Bojesen et al. in a much larger study than our own. ${ }^{12}$

We found that the minor allele of TERC-rs12696304 was associated to a decreased MM risk and with shorter telomere length. This is consistent with the same observation we made for TERT-rs2242652, and it is corroborated by similar findings from a recent study on colorectal cancer. ${ }^{42}$

Longer telomeres might be a marker of an actively reproducing cell that has an increased chance of acquiring tumorcausing mutations. ${ }^{42}$ The association of genetic variability and longer telomere could be of a particular importance in MM since telomerase reactivation and telomerase-mediated elongation of shorter telomeres is a feature of multiple myeloma and because there is an ongoing research on a possible TERT inhibition as a therapeutic approach in $\mathrm{MM}^{42}$

This study has several limitations, first of all we did not use an array to perform the genotyping and we could not take into account ancestry informative markers and therefore we cannot exclude a small proportion of outliers in the population. However it is unlikely that a small number of nonCaucasians would change the results masking a potentially true association or highlighting a spurious one, as suggested by the fact that we replicate the findings by Chubb et al. We have not included all the polymorphic variants present in the regions, even though we have a representation, through tagging, of more than $90 \%$ of the common genetic variability of the TERT and TERC loci. Third, DNA for cases and controls was not extracted from the same starting material since peripheral blood mononuclear cells were used for cases, while whole blood was used for controls.

In summary we have replicated a reported genetic susceptibility locus in TERC, we propose a new one in TERT with an epidemiological and biological explanation, and we describe the association between longer LTL and MM risk. Our data suggest the hypothesis of decreased disease risk by genetic variants that reduce the efficiency of the telomerase complex. This reduced efficiency leads to shorter telomere ends, which in turn are also a marker of decreased MM risk.

\section{Acknowledgements}

The authors acknowledge support by the recruiting hospitals and physicians of the study regions as well as their collaborating nurses and technicians. They thank Ms. Tanja Maihöfer, Mr. Manuel Gentiluomo, Ms. Angelika Stein and Dr. Cosmeri Rizzato (DKFZ, Heidelberg, Germany) for assistance with lab work. 


\section{References}

1. Raab MS, Podar K, Breitkreutz I, et al. Multiple myeloma. Lancet 2009;374:324-39.

2. Martino A, Sainz J, Buda G, et al. Genetics and molecular epidemiology of multiple myeloma: the rationale for the IMMEnSE consortium (review). Int J Oncol 2012;40:625-38.

3. Altieri A, Chen B, Bermejo JL, et al. Familial risks and temporal incidence trends of multiple myeloma. Eur I Cancer 2006;42:1661-70.

4. Landgren O, Weiss BM. Patterns of monoclonal gammopathy of undetermined significance and multiple myeloma in various ethnic/racial groups: support for genetic factors in pathogenesis. Leukemia 2009;23:1691-7.

5. Broderick P, Chubb D, Johnson DC, et al. Common variation at 3 p22.1 and $7 \mathrm{p} 15.3$ influences multiple myeloma risk. Nat Genet 2012;44:58-61.

6. Campa D, Martino A, Sainz J, et al. Comprehensive investigation of genetic variation in the 8q24 region and multiple myeloma risk in the IMMEnSE consortium. Br J Haematol 2012;157: 331-8.

7. Chubb D, Weinhold N, Broderick P, et al. Common variation at $3 \mathrm{q} 26.2,6 \mathrm{p} 21.33,17 \mathrm{p} 11.2$ and 22q13.1 influences multiple myeloma risk. Nat Genet 2013;45:1221-5.

8. Martino A, Campa D, Buda G, et al. Polymorphisms in xenobiotic transporters $A B C B 1$, ABCG2, ABCC2, ABCC1, ABCC3 and multiple myeloma risk: a case-control study in the context of the International Multiple Myeloma rESEarch (IMMEnSE) consortium. Leukemia 2012;26:141922.

9. Martino A, Campa D, Jamroziak K, et al. Impact of polymorphic variation at $7 \mathrm{p} 15.3,3 \mathrm{p} 22.1$ and 2p23.3 loci on risk of multiple myeloma. $\mathrm{Br} \mathrm{J}$ Haematol 2012;158:805-9.

10. Vangsted A, Klausen TW, Vogel U. Genetic variations in multiple myeloma I: effect on risk of multiple myeloma. Eur J Haematol 2012;88:8-30.

11. Weinhold N, Johnson DC, Chubb D, et al. The CCND1 c.870G $>$ A polymorphism is a risk factor for $\mathrm{t}(11 ; 14)(\mathrm{q} 13 ; \mathrm{q} 32)$ multiple myeloma. Nat Genet 2013;45:522-5.

12. Bojesen SE, Pooley KA, Johnatty SE, et al. Multiple independent variants at the TERT locus are associated with telomere length and risks of breast and ovarian cancer. Nat Genet 2013;45: 371-84, 84e1-2.

13. Haiman CA, Chen GK, Vachon CM, et al. A common variant at the TERT-CLPTM1L locus is associated with estrogen receptor-negative breast cancer. Nat Genet 2011;43:1210-4.

14. Kote-Jarai Z, Olama AA, Giles GG, et al. Seven prostate cancer susceptibility loci identified by a multi-stage genome-wide association study. Nat Genet 2011;43:785-91.
15. Kote-Jarai Z, Saunders EJ, Leongamornlert DA, et al. Fine-mapping identifies multiple prostate cancer risk loci at $5 \mathrm{p} 15$, one of which associates with TERT expression. Hum Mol Genet 2013;22: 2520-8.

16. McKay JD, Hung RJ, Gaborieau V, et al. Lung cancer susceptibility locus at $5 \mathrm{p} 15.33$. Nat Genet 2008;40:1404-6.

17. Petersen GM, Amundadottir L, Fuchs CS, et al. A genome-wide association study identifies pancreatic cancer susceptibility loci on chromosomes 13q22.1, 1q32.1 and 5p15.33. Nat Genet 2010;42:224-8.

18. Rajaraman P, Melin BS, Wang Z, et al. Genomewide association study of glioma and meta-analysis. Hum Genet 2012;131:1877-88.

19. Rothman N, Garcia-Closas M, Chatterjee N, et al. A multi-stage genome-wide association study of bladder cancer identifies multiple susceptibility loci. Nat Genet 2010;42:978-84

20. Turnbull C, Rapley EA, Seal S, et al. Variants near DMRT1, TERT and ATF7IP are associated with testicular germ cell cancer. Nat Genet 2010; 42:604-7.

21. Wang Y, Broderick P, Webb E, et al. Common 5 p15.33 and 6 p21.33 variants influence lung cancer risk. Nat Genet 2008;40:1407-9.

22. Fingerlin TE, Murphy E, Zhang W, et al. Genome-wide association study identifies multiple susceptibility loci for pulmonary fibrosis. Nat Genet 2013;45:613-20.

23. Gudmundsson J, Besenbacher S, Sulem P, et al. Genetic correction of PSA values using sequence variants associated with PSA levels. Sci Transl Med 2010;2:62ra92.

24. Mushiroda T, Wattanapokayakit S, Takahashi A, et al. A genome-wide association study identifies an association of a common variant in TERT with susceptibility to idiopathic pulmonary fibrosis. J Med Genet 2008;45:654-6.

25. Blackburn EH. Switching and signaling at the telomere. Cell 2001;106:661-73.

26. Armanios M. Telomeres and age-related disease: how telomere biology informs clinical paradigms. J Clin Investig 2013;123:996-1002.

27. Armanios M, Blackburn EH. The telomere syndromes. Nat Rev Genet 2012;13:693-704.

28. de Lange T. Shelterin: the protein complex that shapes and safeguards human telomeres. Genes Dev 2005;19:2100-10.

29. de Lange T. How telomeres solve the endprotection problem. Science 2009;326:948-52.

30. Martinez P, Blasco MA. Telomeric and extratelomeric roles for telomerase and the telomerebinding proteins. Nat Rev Cancer 2011;11:161-76.

31. McEachern MJ, Krauskopf A, Blackburn EH. Telomeres and their control. Annu Rev Genet 2000;34:331-58.
32. Blasco MA. Telomeres and human disease: ageing, cancer and beyond. Nat Rev Genet 2005;6: 611-22.

33. Wu X, Amos CI, Zhu Y, et al. Telomere dysfunction: a potential cancer predisposition factor. J Natl Cancer Inst 2003;95:1211-8.

34. Chung CC, Chanock SJ. Current status of genome-wide association studies in cancer. Hum Genet 2011;130:59-78.

35. Codd V, Nelson CP, Albrecht E, et al. Identification of seven loci affecting mean telomere length and their association with disease. Nat Genet 2013;45:422-7, 7e1-2.

36. Nan H, Qureshi AA, Prescott J, et al. Genetic variants in telomere-maintaining genes and skin cancer risk. Hum Genet 2011;129:247-53.

37. Roos G, Krober A, Grabowski P, et al. Short telomeres are associated with genetic complexity, high-risk genomic aberrations, and short survival in chronic lymphocytic leukemia. Blood 2008;111: 2246-52.

38. Willeit P, Willeit J, Mayr A, et al. Telomere length and risk of incident cancer and cancer mortality. JAMA 2010;304:69-75.

39. Hosnijeh FS, Matullo G, Russo A, et al. Prediagnostic telomere length and risk of B-cell lymphoma-results from the EPIC cohort study. Int J Cancer J Int Cancer 2014; in press.

40. Kimura M, Gazitt Y, Cao X, et al. Synchrony of telomere length among hematopoietic cells. Exp Hematol 2010;38:854-9.

41. International Myeloma Working Group. Criteria for the classification of monoclonal gammopathies, multiple myeloma and related disorders: a report of the International Myeloma Working Group. Br J Haematol 2003;121:749-57.

42. Jones AM, Beggs AD, Carvajal-Carmona L, et al. TERC polymorphisms are associated both with susceptibility to colorectal cancer and with longer telomeres. Gut 2012;61:248-54.

43. Cawthon RM. Telomere measurement by quantitative PCR. Nucleic Acids Res 2002;30:e47.

44. Gao X, Starmer J, Martin ER. A multiple testing correction method for genetic association studies using correlated single nucleotide polymorphisms Genet Epidemiol 2008;32:361-9.

45. Boyle AP, Hong EL, Hariharan M, et al. Annotation of functional variation in personal genomes using RegulomeDB. Genome Res 2012;22:1790-7.

46. Ward LD, Kellis M. HaploReg: a resource for exploring chromatin states, conservation, and regulatory motif alterations within sets of genetically linked variants. Nucleic Acids Res 2012;40:D9304.

47. Grundberg E, Small KS, Hedman AK, et al. Mapping cis- and trans-regulatory effects across multiple tissues in twins. Nat Genet 2012;44:1084-9. 